\title{
Diabetic kidney disease and pregnancy outcomes: a systematic review
}

\author{
SARAH GLEESON, ${ }^{1,2}$ SHULI SVETITSKY, ${ }^{1}$ CHARLOTTE FRISE 2,3
}

\begin{abstract}
Introduction: We systematically reviewed all relevant literature on diabetic kidney disease (DKD) and pregnancy published in the last 20 years to provide accurate and up-to-date information to inform family planning and maternal care.

Methods: A systematic review was completed in PubMed and Embase. Papers reporting maternal, fetal or renal outcomes of pregnant women with DKD published between 2001 and 2020 were included.

Results: 799 potentially relevant articles were identified, 731 of which were excluded on abstract alone. 68 full-text articles were reviewed and 15 papers were included as they met the selection criteria but were heterogeneous for size, study setting and years studied. The definition of DKD varied between papers and changed over time. 843 women with 873 pregnancies were included. There were high rates of pre-eclampsia and caesarean section, up to $64 \%$ and $100 \%$ respectively. Prematurity and neonatal intensive care admission were common, reported in up to $100 \%$ and $75 \%$, respectively. Maternal and fetal complications were more common with more severe proteinuria and renal impairment. Pregnancy did not hasten progression of DKD.

Discussion: Adverse pregnancy outcomes are frequently encountered and correlate with degree of proteinuria and renal impairment. This information enables individualised risk stratification when a woman is considering pregnancy.

Br J Diabetes 2021;21:175-185
\end{abstract}

Key words: diabetes mellitus, pregnancy, diabetic nephropathy, diabetic kidney disease

\section{Introduction}

Pre-existing diabetes is common, affecting one in every 250 pregnancies, ${ }^{1}$ with diabetic kidney disease (DKD) affecting 2-8\% of those. ${ }^{2}$ Women with diabetes have poorer pregnancy outcomes compared with healthy women; ${ }^{1,3}$ historically, those with DKD have

Renal Department, Imperial College Healthcare NHS Trust, London, UK 2 Obstetric Medicine Department, Imperial College Healthcare NHS Trust, London, UK

Obstetric Medicine Department, Oxford University Hospital, Oxford, UK

Address for correspondence: Dr Sarah Gleeson

Renal Department, Hammersmith Hospital, Imperial College Healthcare

NHS trust, DuCane Road, London, W12 OHS, UK

E-mail: sarah.gleeson7@nhs.net

https://doi.org/10.15277/bjd.2021.306 had even worse outcomes, with fetal mortality rates up to $60 \%{ }^{4}$ More recently, with advances in diabetes management, obstetric and neonatal care, these outcomes have improved, with fetal survival of $95-99 \% .5,6$

Given this relatively high incidence of DKD and the rising prevalence of diabetes, ${ }^{7}$ it is critical to have information on DKD in pregnancy. However, our knowledge of DKD and pregnancy is limited. Much of our information comes from case series and single-centre observational studies, often including small numbers of women, spanning many years. The definition of DKD has also evolved, with earlier studies only concerned with macroalbuminuria and more recent studies including microalbuminuria. ${ }^{5,8}$

We reviewed all relevant literature on DKD and pregnancy published in the last 20 years reporting on maternal, fetal and longer term renal outcomes. This systematic review in a modern timeframe aims to give women considering or entering pregnancy and their healthcare professionals the available information on renal, maternal and fetal risks, to allow them to make informed decisions when family planning and improve care during and after pregnancy.

\section{Methods}

This systematic review was completed in accordance with the Preferred Reporting Items for Systematic Reviews and Metaanalyses (PRISMA). ${ }^{9}$

\section{Search strategy}

We conducted electronic literature searches in PubMed and Embase. The initial search was carried out in August 2020 and repeated in October 2020.The databases were searched for 'diabetic nephropathy', 'diabetic kidney disease', 'microalbuminuria' AND 'pregnancy'. The search was deliberately broad to increase sensitivity. The reference lists of selected papers were searched for references missed by our search strategy.

\section{Selection criteria}

Papers reporting maternal, fetal and/or renal outcomes of pregnant women with DKD published between 2001 and 2020 were included. To reduce publication bias, case reports and series including $\leq 5$ women were excluded. Other exclusion criteria included conference abstracts, papers in languages other than English and pregnancies in women with kidney transplants. If participants were included in more than one report, the larger study was included.

The search was completed in duplicate by SG and SS. They completed the searches independently and matched results. Titles and abstracts were screened by SG and SS. Full texts were assessed by SG. Discrepancies were resolved by discussion. 
Figure 1. Study selection

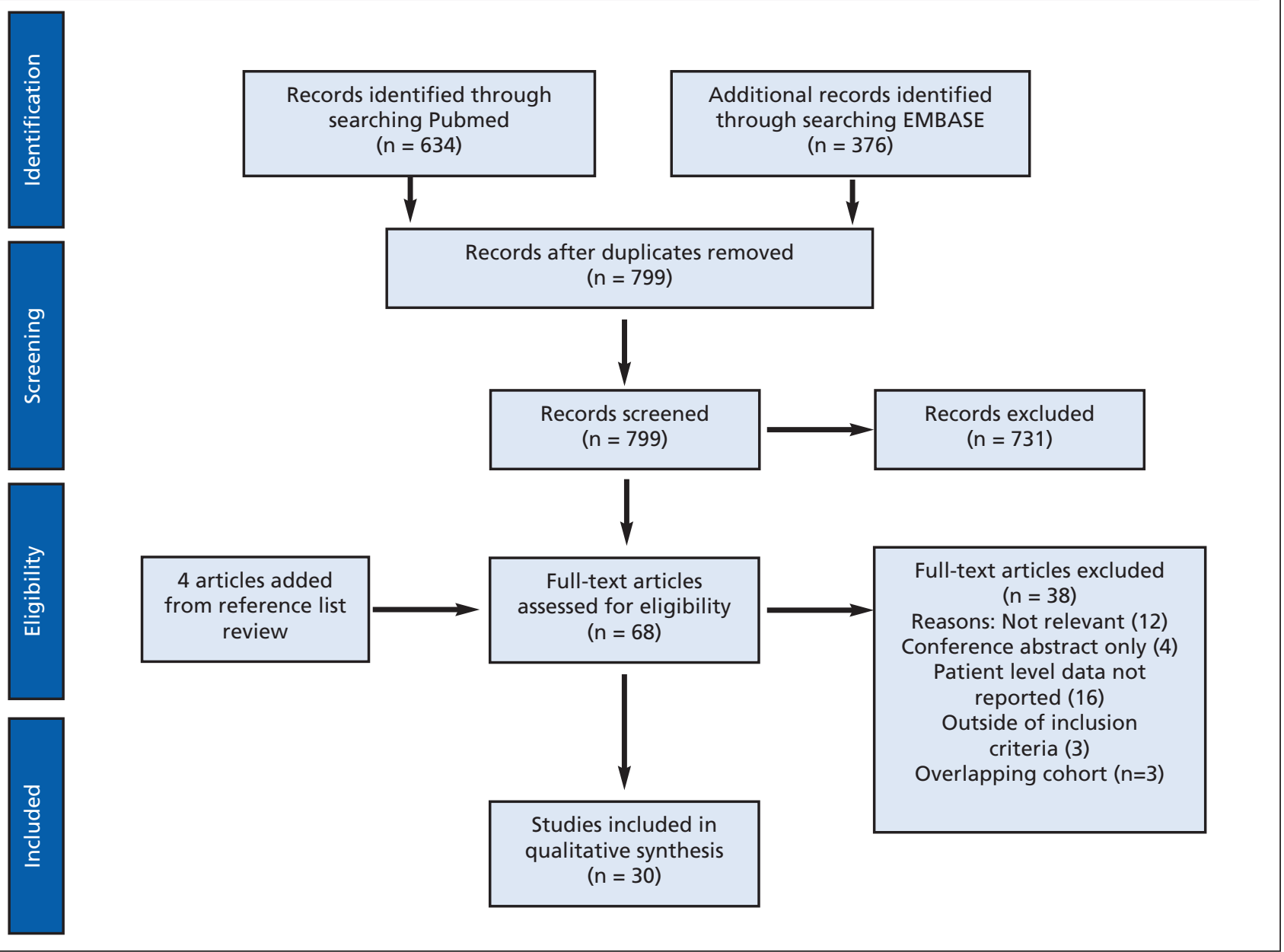

\section{Data collection and analysis}

The data were analysed according to PICOS criteria as follows. The patients $(P)$ were women with DKD. The intervention (I) was considered to be pregnancy, in the absence of an actual therapeutic intervention. The control (C) groups included healthy or women without DKD who were pregnant or women with DKD without pregnancy. The outcomes $(\mathrm{O})$ studied were maternal, fetal and renal outcomes. The studies (S) were all studies reporting on pregnancy outcomes in women with DKD. As the data were expected to be heterogeneous, a narrative review of the results was planned.

Due to the lack of randomised controlled trials and the limited number and variability of control groups, no formal analysis of bias was performed.

\section{Results}

Study selection and general information (Table 1)

A total of 799 potentially relevant articles were identified after excluding duplicates. Of these, 731 were excluded after reviewing the abstract and 68 full-text articles were reviewed. Fifteen papers met the selection criteria and were included (Figure 1), 10 of which were retrospective studies and five were prospec- tive. The studies were heterogeneous for size, study setting and years studied, ranging from 1988 to 2014 . The majority were single-centre studies. Six studies included more than 50 women. The papers were from a range of countries including Denmark, Italy, UK, USA, Brazil, Israel and New Zealand. European countries, in particular Denmark, were the main source of data. Baseline characteristics were often inadequately described and varied between papers. The definitions of DKD varied widely and changed over time, with more recent studies including microalbuminuria (most commonly a urinary albumin of 30-299 mg/ 24 hours) and earlier studies including only 'overt' diabetic nephropathy: macroalbuminuria or macroproteinuria (typically more than 300-500 mg/24 hours proteinuria). One study divided participants into subgroups based on their renal function ${ }^{10}$ and four divided them into subgroups based on micro- or macroalbuminuria. ${ }^{11-14}$ Seven studies included controls, either diabetic or non-diabetic pregnant women or women with DKD who did not have a pregnancy. Study heterogeneity was significant, precluding the pooling of data and meta-analysis.

\section{Baseline characteristics (Table 2)}

Overall, this systematic review collected data on 843 women 
Table 1. General information on studies

\begin{tabular}{|c|c|c|c|c|c|c|c|c|c|}
\hline & Type & Years & Country & Aim & Definitions & Subgroups & Women & Pregnancies & Controls \\
\hline Reece, $1990^{19}$ & Retrospective & 1970-1985 & USA & $\begin{array}{l}\text { To examine the effect of } \\
\text { pregnancy on the rate of } \\
\text { progression of DN }\end{array}$ & $\begin{array}{l}300 \mathrm{mg} / \text { day prior to } \\
\text { 3rd trimester }\end{array}$ & NA & 10 & 11 & NA \\
\hline Combs, $1993^{20}$ & Retrospective & 1982-1991 & USA & $\begin{array}{l}\text { To examine if pre-eclampsia in } \\
\text { diabetic mothers is increased } \\
\text { in incipient as well as overt } \\
\text { nephropathy }\end{array}$ & $\begin{array}{l}>500 \mathrm{mg} \text { proteinuria/ } \\
\text { day }\end{array}$ & NA & 62 & 62 & $\begin{array}{l}\text { No nephropathy } \\
\text { Proteinuria 190- } \\
499 \text { mg/24h }\end{array}$ \\
\hline Hod, $1995^{21}$ & Prospective & 1990-1993 & Israel & $\begin{array}{l}\text { To examine whether } \\
\text { treatment with ACE inhibitor } \\
\text { pre-pregnancy improves } \\
\text { pregnancy outcomes }\end{array}$ & $\begin{array}{l}>500 \mathrm{mg} \text { proteinuria/ } \\
\text { day }\end{array}$ & NA & 8 & 8 & NA \\
\hline Kimmerle, $1995^{4}$ & Retrospective & 1982-1992 & Germany & $\begin{array}{l}\text { To study the effect of DKD on } \\
\text { pregnancy and perinatal } \\
\text { outcome, infant development } \\
\text { and long-term function }\end{array}$ & $\begin{array}{l}>400 \mathrm{mg} \text { proteinuria/ } \\
24 \mathrm{~h}\end{array}$ & $\begin{array}{l}\text { Preserved renal function } \\
(\mathrm{CrCl}>80 \mathrm{~mL} / \mathrm{min}) \\
\text { Without preserved renal } \\
\text { function }(\mathrm{CrCl}<80 \mathrm{~mL} / \mathrm{min})\end{array}$ & $\begin{array}{l}\text { Overall } \\
\text { cohort } \\
33\end{array}$ & $\begin{array}{l}\text { Overall } \\
\text { cohort } \\
40\end{array}$ & $\begin{array}{l}110 \text { in diabetic } \\
\text { women without } \\
\text { nephropathy }\end{array}$ \\
\hline Gordon, $1996^{22}$ & Retrospective & 1988-1994 & USA & $\begin{array}{l}\text { To determine outcomes in } \\
\text { pregnancies complicated by } \\
\text { DN (white class F) }\end{array}$ & $\begin{array}{l}>400 \mathrm{mg} / 24 \mathrm{~h} \text { or } \mathrm{CrCl} \\
<90 \mathrm{~mL} / \mathrm{min}\end{array}$ & NA & 51 & 51 & NA \\
\hline Kaаja, $1996^{23}$ & Prospective & 1983-1985 & Finland & $\begin{array}{l}\text { To establish whether } \\
\text { pregnancy affects long-term } \\
\text { development and progression } \\
\text { of retinopathy and } \\
\text { nephropathy in diabetic } \\
\text { women }\end{array}$ & $\begin{array}{l}\text { White class } \mathrm{F}(\mathrm{CrCl} \\
>100 \mathrm{~mL} / \mathrm{min}, \\
\text { creatinine }<90 \text { umol/L }\end{array}$ & NA & 6 & 9 & $\begin{array}{l}4 \text { women with DN } \\
\text { without pregnancy }\end{array}$ \\
\hline Mackie, $1996^{10}$ & Retrospective & 1985-1993 & UK & $\begin{array}{l}\text { To examine the effect of } \\
\text { pregnancy on maternal renal } \\
\text { function in women with DN }\end{array}$ & $>500 \mathrm{mg} / 24 \mathrm{~h}$ protein & $\begin{array}{l}\text { Moderate renal } \\
\text { impairment } \\
\text { (serum creatinine } \\
>125 \mathrm{mmol} / \mathrm{L} \text { ) } \\
\text { Mild renal impairment } \\
\text { (serum creatinine } \\
<125 \mathrm{mmol} / \mathrm{L} \text { ) }\end{array}$ & 12 & 11 & NA \\
\hline Miodovnik, $1996^{24}$ & Prospective & 1978-1991 & USA & $\begin{array}{l}\text { To examine whether } \\
\text { pregnancy increases the } \\
\text { risk of or accelerates the } \\
\text { progression of DN }\end{array}$ & $\begin{array}{l}>500 \mathrm{mg} / \text { day } \\
\text { proteinuria }\end{array}$ & NA & 56 & 56 & $\begin{array}{l}\text { Diabetic pregnant } \\
\text { women without } \\
\text { nephropathy }\end{array}$ \\
\hline Purdy, $1996^{25}$ & Retrospective & 1981-1993 & USA & $\begin{array}{l}\text { To determine whether } \\
\text { pregnancy worsens renal } \\
\text { function in women with } \\
\text { DN and moderate-to-severe } \\
\text { renal insufficiency }\end{array}$ & $\begin{array}{l}\text { Serum creatinine } \\
>124 \mathrm{mmol} / \mathrm{L}\end{array}$ & NA & 11 & 11 & $\begin{array}{l}11 \text { women with } \\
\text { similar renal } \\
\text { function without } \\
\text { pregnancy }\end{array}$ \\
\hline Zhu, $1997^{26}$ & Retrospective & 1984-1996 & Japan & $\begin{array}{l}\text { To evaluate the outcomes of } \\
\text { pregnancies complicated with } \\
\text { diabetes mellitus }\end{array}$ & NR & NA & 10 & 10 & $\begin{array}{l}\text { Pregnancies in } \\
\text { women with } \\
\text { diabetes without } \\
\text { nephropathy }\end{array}$ \\
\hline Reece, $1998^{27}$ & Retrospective & 1984-1990 & USA & $\begin{array}{l}\text { To report their } 10 \text {-year } \\
\text { experience in caring for } \\
\text { patients with DN }\end{array}$ & $\begin{array}{l}>300 \mathrm{mg} \text { albumin or } \\
\text { protein/24h }\end{array}$ & NA & 27 & 27 & NA \\
\hline Bar, $1999^{28}$ & Prospective & 1990-1995 & Israel & $\begin{array}{l}\text { To examine the effect of } \\
\text { pre-pregnancy captopril on } \\
\text { renal function and on fetal- } \\
\text { maternal outcome in DN }\end{array}$ & $\begin{array}{l}\text { Proteinuria }>500 \\
\mathrm{mg} / \text { day }\end{array}$ & NA & 24 & 24 & NA \\
\hline Biesenbach, $1999^{11}$ & Retrospective & 1982-1996 & Austria & $\begin{array}{l}\text { To evaluate the impact of } \\
\text { pregnancy on the course of } \\
\text { renal function in women } \\
\text { with overt DN }\end{array}$ & $\begin{array}{l}\text { Macroproteinuria } \\
>0.5 \mathrm{~g} \text { protein- } \\
\text { uria/24h }\end{array}$ & $\begin{array}{l}\text { Increase in creatinine } \\
\text { clearance during } \\
\text { 1st two trimesters of } \\
\text { pregnancy } \\
\text { No increase in } \mathrm{CrCl}\end{array}$ & $\begin{array}{l}12 \\
5 \\
7\end{array}$ & $\begin{array}{l}14 \\
6 \\
8\end{array}$ & NA \\
\hline Dunne, $1999^{29}$ & Retrospective & 1990-1997 & UK & $\begin{array}{l}\text { To examine fetal/maternal } \\
\text { outcomes in women with DN }\end{array}$ & $\begin{array}{l}>300 \mathrm{mg} / 24 \mathrm{~h} \text { or } \\
>1+\times 3\end{array}$ & NA & 18 & 21 & NA \\
\hline Biesenbach, $2000^{30}$ & Retrospective & 1985-1993 & Austria & $\begin{array}{l}\text { To evaluate perinatal } \\
\text { complications and follow-up } \\
\text { of infants of mothers with } \\
\text { DN stage IV }\end{array}$ & $\begin{array}{l}500 \mathrm{mg} / 24 \mathrm{~h} \\
\text { proteinuria }\end{array}$ & NA & 10 & 10 & NA \\
\hline Ekbom, $2001^{14}$ & Prospective & 1996-2000 & Denmark & $\begin{array}{l}\text { Pregnancy outcome in T1 } \\
\text { diabetic women with } \\
\text { microalbuminuria }\end{array}$ & $\begin{array}{l}\text { DKD } \\
>300 \text { mg/24h } \\
\text { Microalbuminuria } \\
30-300 \text { mg/24h }\end{array}$ & $\begin{array}{l}\text { Microalbuminuria } \\
\text { DN }\end{array}$ & $\begin{array}{l}26 \\
11\end{array}$ & $\begin{array}{l}26 \\
11\end{array}$ & $\begin{array}{l}\text { Diabetic women } \\
\text { with no } \\
\text { microalbuminuria }\end{array}$ \\
\hline
\end{tabular}


Table 1. General information on studies (continued)

\begin{tabular}{|c|c|c|c|c|c|c|c|c|c|}
\hline & Type & Years & Country & Aim & Definitions & Subgroups & Women & Pregnancies & Controls \\
\hline Khoury, $2002^{9}$ & Retrospective & NR & USA & $\begin{array}{l}\text { To examine the association of } \\
\text { renal function with maternal } \\
\text { and fetal pregnancy outcome } \\
\text { in women with DN }\end{array}$ & $\begin{array}{l}\text { DN: proteinuria } \\
>500 \mathrm{mg} / 24 \mathrm{~h}\end{array}$ & $\begin{array}{l}\mathrm{Cr}<1 \mathrm{mg} / \mathrm{dL} \\
\mathrm{Cr} 1-1.5 \mathrm{mg} / \mathrm{dL} \\
\mathrm{Cr}>1.5 \mathrm{mg} / \mathrm{dL}\end{array}$ & $\begin{array}{l}58 \text { (total } \\
\text { cohort) }\end{array}$ & $\begin{array}{l}72 \text { (total } \\
\text { cohort) } \\
49 \\
13 \\
10\end{array}$ & NA \\
\hline Rossing, $2002^{18}$ & Retrospective & 1970-1989 & Denmark & $\begin{array}{l}\text { To examine the long-term } \\
\text { impact of pregnancy on the } \\
\text { progression of DN }\end{array}$ & $\begin{array}{l}\text { Albuminuria }>300 \\
\mathrm{mg} / 24 \mathrm{~h}\end{array}$ & NA & 26 & 31 & $\begin{array}{l}67 \text { women without } \\
\text { pregnancies }\end{array}$ \\
\hline Bagg, $2003^{31}$ & Prospective & 1985-2000 & $\begin{array}{l}\text { New } \\
\text { Zealand }\end{array}$ & $\begin{array}{l}\text { To describe long-term } \\
\text { maternal outcome after } \\
\text { pregnancy in women with DN }\end{array}$ & $\begin{array}{l}>300 \mathrm{mg} / 24 \mathrm{~h} \\
\text { albuminuria }\end{array}$ & NA & 14 & 24 & NA \\
\hline Carr, 20067 & Retrospective & 1986-2002 & USA & $\begin{array}{l}\text { To evaluate if hypertension in } \\
\text { early pregnancy is associated } \\
\text { with adverse perinatal } \\
\text { outcome in women with DN }\end{array}$ & $\begin{array}{l}\text { Proteinuria }>0.3 \\
\mathrm{~g} / 24 \mathrm{~h}\end{array}$ & $\begin{array}{l}\text { Above target BP (MAP } \\
>100 \mathrm{mmHg} \text { ) } \\
\text { Below target (MAP } \\
<100 \mathrm{mmHg} \text { ) }\end{array}$ & $\begin{array}{l}43 \\
22 \\
21\end{array}$ & $\begin{array}{l}43 \\
22 \\
21\end{array}$ & NA \\
\hline Nielson, $2006^{32}$ & Retrospective & 1995-2003 & Denmark & $\begin{array}{l}\text { To describe the impact of } \\
\text { aggressive antihypertensive } \\
\text { treatment in the prevalence } \\
\text { of preterm delivery in women } \\
\text { with DM }\end{array}$ & $\begin{array}{l}\text { Albuminuria } 30-300 \\
\mathrm{mg} / 24 \mathrm{~h}\end{array}$ & $\begin{array}{l}1995-1999 \\
2000-2003\end{array}$ & $\begin{array}{l}26 \\
20\end{array}$ & $\begin{array}{l}26 \\
20\end{array}$ & NA \\
\hline Nielsen, $2009^{12}$ & Prospective & 2004-2006 & Denmark & $\begin{array}{l}\text { To describe outcomes in } \\
\text { microalbuminuria or DN after } \\
\text { intensified anti-hypertensive } \\
\text { therapy }\end{array}$ & $\begin{array}{l}\text { DN: } \\
>300 \mathrm{mg} \\
\text { albumin/24h } \\
\text { Microalbuminuria: } \\
30-299 \mathrm{mg} \\
\text { albumin/24h }\end{array}$ & $\begin{array}{l}\text { DN } \\
\text { Microalbuminuria }\end{array}$ & $\begin{array}{l}7 \\
10\end{array}$ & $\begin{array}{l}7 \\
10\end{array}$ & $\begin{array}{l}100 \text { women with } \\
\text { normoalbuminuria } \\
25 \text { healthy } \\
\text { pregnant women }\end{array}$ \\
\hline Yogev, $2009^{33}$ & Retrospective & 2000-2007 & Israel & $\begin{array}{l}\text { To examine the factors } \\
\text { associated with pregnancy } \\
\text { complications in women with } \\
\text { type } 1 \text { diabetes and DN }\end{array}$ & $\begin{array}{l}\text { Protein } 300 \mathrm{mg} / \\
24 \mathrm{~h} \text { pre or early } \\
\text { pregnancy or serum } \\
\text { creatinine }>1.5\end{array}$ & $\begin{array}{l}\text { Non-complicated } \\
\text { pregnancy } \\
\text { Complicated pregnancy }\end{array}$ & $\begin{array}{l}15 \\
31\end{array}$ & $\begin{array}{l}15 \\
31\end{array}$ & NA \\
\hline Jensen, $2010^{34}$ & Prospective & 1993-1999 & Denmark & $\begin{array}{l}\text { To describe microalbuminuria, } \\
\text { pre-eclampsia, and preterm } \\
\text { delivery in pregnant women } \\
\text { with type } 1 \text { diabetes on a } \\
\text { national level }\end{array}$ & $\begin{array}{l}\text { Albuminuria } 30-300 \\
\mathrm{mg} / 24 \mathrm{~h}\end{array}$ & NA & 84 & 84 & $\begin{array}{l}\text { Pregnant diabetic } \\
\text { women without } \\
\text { albuminuria }\end{array}$ \\
\hline Bell, $2012^{17}$ & $\begin{array}{l}\text { Population- } \\
\text { based cohort }\end{array}$ & 1996-2008 & UK & $\begin{array}{l}\text { To quantify the risk of major } \\
\text { congenital anomaly and to } \\
\text { assess the influence of various } \\
\text { risk factors including DN }\end{array}$ & Not reported & NA & 60 & 60 & $\begin{array}{l}\text { Women with } \\
\text { pregnancies } \\
\text { complicated by } \\
\text { congenital } \\
\text { malformations } \\
\text { without DN }\end{array}$ \\
\hline Young, $2012^{35}$ & Prospective & 2010-2011 & Brazil & $\begin{array}{l}\text { To examine the effect of } \\
\text { pregnancy on } \mathrm{DN} \text { and the } \\
\text { perinatal outcomes of } \\
\text { diabetic pregnancies }\end{array}$ & $\begin{array}{l}\text { Albuminuria }>30 \\
\mathrm{mg} / 24 \mathrm{~h}\end{array}$ & NA & 11 & 11 & $\begin{array}{l}32 \\
\text { pregnancies in } \\
\text { diabetic women } \\
\text { without DN }\end{array}$ \\
\hline Damm, $2013^{5}$ & Retrospective & 2007-2012 & Denmark & $\begin{array}{l}\text { To evaluate the prevalence of } \\
\text { DN and microalbuminuria in } \\
\text { pregnant women with type } 2 \\
\text { diabetes in comparison with } \\
\text { type } 1 \text { diabetes and to } \\
\text { describe pregnancy outcomes }\end{array}$ & $\begin{array}{l}\text { Nephropathy: } \\
\text { ACR >300 mg/g } \\
\text { Microalbuminuria: } \\
\text { ACR 30-299 mg/g }\end{array}$ & $\begin{array}{l}\text { T2 nephropathy } \\
\text { T1 nephropathy } \\
\text { T2 microalbuminuria } \\
\text { T1 microalbuminuria }\end{array}$ & $\begin{array}{l}5 \\
11 \\
10 \\
15\end{array}$ & $\begin{array}{l}5 \\
11 \\
10 \\
15\end{array}$ & NA \\
\hline Piccoli, $2013^{15}$ & Retrospective & 2000-2012 & Italy & $\begin{array}{l}\text { To evaluate maternal and } \\
\text { fetal outcomes in severe DN }\end{array}$ & $\begin{array}{l}\text { Severe nephropathy: } \\
\text { referred to } \\
\text { nephrology clinic } \\
\text { from diabetes in } \\
\text { pregnancy clinic }\end{array}$ & NA & 11 & 12 & NA \\
\hline Klemetti, $2015^{16}$ & Retrospective & 1988-2011 & Finland & $\begin{array}{l}\text { To analyse temporal changes } \\
\text { in the glycaemic control, BP } \\
\text { levels, markers of renal } \\
\text { function as and perinatal out- } \\
\text { comes of a population-based } \\
\text { cohort of women with DN }\end{array}$ & $\begin{array}{l}\text { Proteinuria }>0.3 \\
\text { g/24h or dipstick } 1+\end{array}$ & $\begin{array}{l}1988-1999 \\
2000-2011\end{array}$ & $\begin{array}{l}65 \\
43\end{array}$ & $\begin{array}{l}65 \\
43\end{array}$ & NA \\
\hline Seah, $2020^{13}$ & Retrospective & 2004-2014 & Australia & $\begin{array}{l}\text { Association between } \\
\text { maternal renal function and } \\
\text { pregnancy outcomes in } \\
\text { type } 1 \text { and type } 2 \text { diabetes }\end{array}$ & $\begin{array}{l}\text { Microalbuminuria: } \\
3-300 \mathrm{mg} / \text { day or } \\
\text { ACR of 3.4-35 } \\
\text { Macroalbuminuria: } \\
>300 \mathrm{mg} / \text { day or } \\
\text { ACR }>35\end{array}$ & $\begin{array}{l}\text { Microalbuminuria } \\
\text { Macroalbuminuria }\end{array}$ & $\begin{array}{l}198 \text { with } \\
\text { diabetes } \\
\text { Number } \\
\text { with } \\
\text { nephro- } \\
\text { pathy NR }\end{array}$ & & $\begin{array}{l}119 \text { pregnancies in } \\
\text { healthy women }\end{array}$ \\
\hline
\end{tabular}


Table 2 Baseline characteristics

\begin{tabular}{|c|c|c|c|c|c|c|c|c|c|c|c|}
\hline & Age & Ethnicity & $\begin{array}{l}\text { Duration } \\
\text { of diabetes } \\
\text { (years) }\end{array}$ & $\begin{array}{l}\text { Hypertension } \\
(\%)\end{array}$ & $\begin{array}{l}\text { Retinopathy } \\
(\%)\end{array}$ & $\begin{array}{l}\text { Baseline } \\
\text { creatinine }\end{array}$ & $\begin{array}{l}\text { Type of } \\
\text { diabetes }\end{array}$ & $\begin{array}{l}\text { Baseline } \\
\mathrm{HbA}_{1 \mathrm{c}} \\
(\%)\end{array}$ & $\begin{array}{l}\text { Baseline } \\
\text { proteinuria }\end{array}$ & $\begin{array}{l}\text { Baseline } \\
\text { eGFR (ml/min) } \\
\text { or CrCl (ml/min) }\end{array}$ & $\begin{array}{l}\text { Nulliparity } \\
(\%)\end{array}$ \\
\hline Reece, $1990^{19}$ & 30 & NR & NR & 91 & 100 & $1.3 \mathrm{mg} / \mathrm{dL}$ & & & $2.5 \mathrm{~g} / 24 \mathrm{~h}$ & NR & NR \\
\hline Combs, $1993^{20}$ & 27.3 & NR & 14.3 & 39 & 37 & 0.91 & T1 & 9.0 & NR & 56 & NR \\
\hline Hod, $1995^{21}$ & 25.6 & NR & 15.6 & NR & 37.5 & $0.8 \mathrm{mg} / \mathrm{dL}$ & $\mathrm{T} 1$ & 7.9 & $273 \mathrm{mg} / 24 \mathrm{~h}$ & 114 & NR \\
\hline Kimmerle, $1995^{4}$ & 29 & NR & 20 & 61 & 65 & NR & NR & NR & $2.1 \mathrm{~g} / 24 \mathrm{~h}$ & NR & NR \\
\hline Gordon, $1996^{22}$ & 25.5 & $76 \%$ white & 15 & 27 & 53 & 0.8 & $\mathrm{~T} 1$ & NR & $1.74 \mathrm{~g} / 24$ & 120 & 64 \\
\hline Kaaja, $1996^{23}$ & 35.5 & & 21.7 & $11 \%$ & NR & NR & NR & NR & NR & NR & NR \\
\hline $\begin{array}{l}\text { Mackie, } 1996^{10} \\
\text { Moderate renal } \\
\text { impairment }\end{array}$ & 30.5 & NR & 17 & 16.6 & NR & 160 & NR & NR & $3.8 \mathrm{~g} / 24 \mathrm{~h}$ & NR & NR \\
\hline $\begin{array}{l}\text { Mild renal } \\
\text { impairment }\end{array}$ & NR & & NR & NR & & $N R$ & & & NR & & \\
\hline Miodovnik, $1996^{24}$ & 25.5 & NR & 14.7 & 40.8 & 39.2 & NR & NR & $9.8 \%$ & NR & NR & 32 \\
\hline Purdy, $1996^{25}$ & 29 & Mainly white & 20 & NR & NR & 159 & NR & NR & $2.4 \mathrm{~g} / 24 \mathrm{~h}$ & NR & NR \\
\hline Zhu, $1997^{26}$ & NR & NR & NR & NR & NR & NR & NR & NR & NR & NR & NR \\
\hline Reece, $1998^{27}$ & 27 & & 16.4 & 77 & 89 & NR & NR & NR & NR & NR & NR \\
\hline Bar, $1999^{28}$ & 26 & NR & NR & 46 & 37.5 & $0.82 \mathrm{mg} / \mathrm{dL}$ & $\mathrm{T} 1$ & 7.9 & $202 \mathrm{mg} / 24 \mathrm{~h}$ & NR & NR \\
\hline Biesenbach, $1999^{11}$ & $\begin{array}{l}29 \\
28 \\
29\end{array}$ & $\begin{array}{l}\text { NR } \\
\text { NR }\end{array}$ & $\begin{array}{l}18 \\
17 \\
20\end{array}$ & NR & NR & $\begin{array}{l}111 \\
96 \\
122\end{array}$ & NR & $\begin{array}{l}8.0 \\
8 \\
8\end{array}$ & $\begin{array}{l}1.7 \\
1.1 \mathrm{~g} / 24 \mathrm{~h} \\
2.2 \mathrm{~g} / 24 \mathrm{~h}\end{array}$ & $\begin{array}{l}69 \\
80 \\
61\end{array}$ & NR \\
\hline Dunne, $1999^{29}$ & 26.5 & NR & 19.5 & 11 & NR & 88.3 & $\mathrm{~T} 1$ & 9.7 & NR & NR & NR \\
\hline Biesenbach, $2000^{30}$ & NR & NR & NR & NR & NR & $N R$ & NR & NR & NR & NR & NR \\
\hline $\begin{array}{l}\text { Ekbom, } 2001^{14} \\
\text { DN } \\
\text { Microalbuminuria }\end{array}$ & $\begin{array}{l}29 \\
30\end{array}$ & NR & $\begin{array}{l}19 \\
16\end{array}$ & NR & $\begin{array}{l}77 \\
100\end{array}$ & NR & $\mathrm{T} 1$ & $\begin{array}{l}8.1 \\
8.8\end{array}$ & $\begin{array}{l}69 \mathrm{mg} / 24 \mathrm{~h} \\
1120\end{array}$ & NR & $\begin{array}{l}50 \\
55\end{array}$ \\
\hline $\begin{array}{l}\text { Khoury, } 2002^{9} \\
\mathrm{Cr}<1 \mathrm{mg} / \mathrm{dL} \\
\mathrm{Cr} 1-1.5 \mathrm{mg} / \mathrm{dL} \\
\mathrm{Cr}>1.5 \mathrm{mg} / \mathrm{dL}\end{array}$ & $\begin{array}{l}26.3 \\
28.3 \\
29.0\end{array}$ & $\begin{array}{l}14.3 \% \text { black } \\
0 \% \text { black } \\
30 \% \text { black }\end{array}$ & $\begin{array}{l}15.4 \\
16.5 \\
15.6\end{array}$ & $\begin{array}{l}12.2 \\
69.2 \\
90\end{array}$ & $\begin{array}{l}24.5 \\
46.2 \\
80\end{array}$ & NR & $\begin{array}{l}\text { T1 } \\
\text { T1 } \\
\text { T1 }\end{array}$ & $\begin{array}{l}9.9 \\
9.5 \\
8.9\end{array}$ & $\begin{array}{l}800 \mathrm{mg} / 24 \mathrm{~h} \\
1796 \\
1606\end{array}$ & $\begin{array}{l}87.8 \mathrm{~mL} / \mathrm{min} \\
79.2 \\
41.5\end{array}$ & $\begin{array}{l}51 \\
61.5 \\
60\end{array}$ \\
\hline Rossing, $2002^{18}$ & 24 & NR & 14 & NR & NR & $79 \mathrm{mmol} / \mathrm{L}$ & $\mathrm{T} 1$ & NR & $534 \mathrm{mg} / 24 \mathrm{~h}$ & NR & NR \\
\hline Bagg, $2003^{31}$ & 30 & NR & 18.5 & NR & NR & $0.07 \mathrm{mmol} / \mathrm{L}$ & $\mathrm{T} 1$ and $\mathrm{T} 2$ & NR & NR & NR & NR \\
\hline $\begin{array}{l}\text { Carr, } 2006^{7} \\
\text { Above target BP } \\
\text { (MAP > } 100 \mathrm{mmHg})\end{array}$ & 29.5 & & 16 & 59.1 & 63.6 & $0.85 \mathrm{mg} / \mathrm{dL}$ & $\mathrm{T} 1$ & 8.1 & $1.65 \mathrm{~g} / 24 \mathrm{~h}$ & $135.9 \mathrm{~mL} / \mathrm{min}$ & NR \\
\hline $\begin{array}{l}\text { Below target } \\
\text { (MAP <100 mmHg) }\end{array}$ & 27.2 & & 17.5 & 85.7 & 85.7 & $1.23 \mathrm{mg} / \mathrm{dL}$ & & 8 & 4.69 & $90.2 \mathrm{~mL} / \mathrm{min}$ & \\
\hline $\begin{array}{l}\text { Nielson, } 2006^{32} \\
1995-1999 \\
2000-2003\end{array}$ & $\begin{array}{l}19 \\
18\end{array}$ & NR & $\begin{array}{l}6.7 \\
6.8\end{array}$ & NR & NR & NR & $\mathrm{T} 1$ & NR & $\begin{array}{l}69 \mathrm{mg} / 24 \mathrm{~h} \\
74\end{array}$ & NR & NR \\
\hline $\begin{array}{l}\text { Nielsen, } 2009^{12} \\
\text { Diabetic nephropathy } \\
\text { Microalbuminuria }\end{array}$ & $\begin{array}{l}30 \\
31\end{array}$ & NR & $\begin{array}{l}20 \\
14\end{array}$ & $\begin{array}{l}100 \\
50\end{array}$ & $\begin{array}{l}100 \\
50\end{array}$ & $\begin{array}{l}57 \\
51\end{array}$ & $\mathrm{~T} 1$ & $\begin{array}{l}6.5 \\
6.9\end{array}$ & $\begin{array}{l}690 \mathrm{mg} / 24 \mathrm{~h} \\
91\end{array}$ & NR & NR \\
\hline $\begin{array}{l}\text { Yogev, } 2009^{33} \\
\text { Non-complicated } \\
\text { pregnancy } \\
\text { Complicated } \\
\text { pregnancy }\end{array}$ & $\begin{array}{l}31.8 \\
31.2\end{array}$ & NR & $\begin{array}{l}18 \\
19.7\end{array}$ & $\begin{array}{l}80 \\
89\end{array}$ & $\begin{array}{l}53 \\
32\end{array}$ & $\begin{array}{l}1.08 \\
1.11\end{array}$ & $\mathrm{~T} 1$ & $\begin{array}{l}7.1 \\
7.5\end{array}$ & $\begin{array}{l}53 \% \text { none, } 47 \% \\
<20 \mathrm{mg} / 24 \mathrm{~h} \\
74 \% \mathrm{none}, 13 \% \\
<20 \mathrm{mg} / 24 \mathrm{~h}, 6.5 \% \\
20-300 \mathrm{mg} / 24 \mathrm{~h}, 6.5 \% \\
>300 \mathrm{mg} / 24 \mathrm{~h}\end{array}$ & $\%$ & NR \\
\hline Jensen, $2010^{34}$ & 27 & NR & 15 & 13 & 11 & NR & $\mathrm{T} 1$ & 7.6 & NR & 68 & NR \\
\hline Bell, $2012^{17}$ & NR & NR & NR & NR & NR & NR & NR & NR & NR & NR & NR \\
\hline Young, $2012^{35}$ & 28.3 & 45\% Caucasian & n 12 & 72.7 & 54.6 & $0.8 \mathrm{mg} / \mathrm{dL}$ & $81.2 \% \mathrm{~T} 1$ & 8.5 & $119 \mathrm{mg} / 24 \mathrm{~h}$ & $81 \mathrm{~mL} / \mathrm{min}$ & $27 \%$ \\
\hline $\begin{array}{l}\text { Damm, } 2013^{5} \\
\text { Type } 2 \text { DN } \\
\text { Type } 1 \text { DN } \\
\text { T2 microalbuminuria } \\
\text { T1 microalbuminuria }\end{array}$ & $\begin{array}{l}31 \\
32 \\
31 \\
31\end{array}$ & NR & $\begin{array}{l}2 \\
19 \\
2 \\
22\end{array}$ & $\begin{array}{l}0 \\
64 \\
0 \\
60\end{array}$ & $\begin{array}{l}75 \\
56 \\
20 \\
85\end{array}$ & $\begin{array}{l}52 \\
61 \\
40 \\
51\end{array}$ & $\begin{array}{l}\mathrm{T} 2 \\
\mathrm{~T} 1 \\
\mathrm{~T} 2 \\
\mathrm{~T} 1\end{array}$ & $\begin{array}{l}6.8 \\
7 \\
6.8 \\
7.1\end{array}$ & $\begin{array}{l}\text { ACR } \\
474 \mathrm{mg} / \mathrm{mol} \\
712 \\
110 \\
84.5\end{array}$ & NR & $\begin{array}{l}50 \\
45 \\
30 \\
67\end{array}$ \\
\hline Piccoli, $2013^{15}$ & 34.3 & NR & 22.6 & $66 \%$ & $100 \%$ & $0.98 \mathrm{mg} / \mathrm{dL}$ & $\mathrm{T} 1$ & $8.01 \%$ & $1.6 \mathrm{~g} / 24 \mathrm{~h}$ & $67 \mathrm{~mL} / \mathrm{min}$ & NR \\
\hline $\begin{array}{l}\text { Klemetti, } 2015^{16} \\
\text { 1988-1999 group } \\
\text { 2000-2011 group }\end{array}$ & $\begin{array}{l}29 \\
31\end{array}$ & NR & $\begin{array}{l}19 \\
24\end{array}$ & $\begin{array}{l}34.4 \\
65.1\end{array}$ & $\begin{array}{l}50.8 \\
65.1\end{array}$ & $\begin{array}{l}82 \mu \mathrm{mol} / \mathrm{L} \\
68 \mu \mathrm{mol} / \mathrm{L}\end{array}$ & $\begin{array}{l}\text { T1 } \\
\text { T1 }\end{array}$ & $\begin{array}{l}66 \mathrm{mmo} / \mathrm{mol} \\
69\end{array}$ & $\begin{array}{l}1.5 \mathrm{~g} / 24 \mathrm{~h} \\
0.8\end{array}$ & $\begin{array}{l}1.12 \\
1.74\end{array}$ & $\begin{array}{l}46.2 \\
60.5\end{array}$ \\
\hline Seah, $2020^{13}$ & NR & NR & NR & NR & NR & NR & NR & NR & NR & NR & NR \\
\hline
\end{tabular}

DN, diabetic nephropathy; $C r$, creatinine; NR, not reported. 
with DKD experiencing 873 pregnancies. The mean age ranged from 24 to 34 years and the mean duration of diabetes ranged from 2 years (in two subgroups with type 2 diabetes) ${ }^{11}$ to 22.6 years. ${ }^{15}$ Where reported, both pre-pregnancy hypertension and retinopathy ranged from $11 \%$ in a cohort with microalbuminuria to $100 \%$ in women with overt proteinuria. Across the studies, $27-67 \%$ of women were nulliparous. Values for baseline creatinine, estimated glomerular filtration rate (eGFR) or creatinine clearance, proteinuria or albuminuria and $\mathrm{HBA}_{1 c}$ were given either pre-pregnancy or in early pregnancy. One paper ${ }^{16}$ divided its study participants into subgroups based on whether they had a complicated or uncomplicated pregnancy. These results are included in Tables 1-5 but have been excluded from the analysis below.

\section{Maternal outcomes (Table 3)}

There were high rates of pre-eclampsia and caesarean section, especially in those with impaired renal function, more severe proteinuria or both. Pre-eclampsia was commonly reported, ranging from $0 \%{ }^{12}$ in one subgroup of 10 women with microalbuminuria to $64 \%$ (IQR $33.3-42.5 \%) ;{ }^{17}$ compared to healthy women, women with diabetic kidney disease were more likely to develop pre-eclampsia (OR 5.5 (2.5 to 11.8)). ${ }^{13}$ One study which included diabetic women without albuminuria, with microalbuminuria and macroalbuminuria reported pre-eclampsia in $6 \%, 42 \%$ and $64 \%$, respectively. ${ }^{14}$ Caesarean section was the most common method of delivery, ranging from $20 \%$ to $100 \%$ (IQR $69.2-$ 90.0). No papers reported maternal death. One paper reported requirement for renal replacement therapy in one of 108 pregnancies. ${ }^{18}$

\section{Fetal outcomes (Table 4)}

The mean gestational age ranged from 32.5 weeks in a cohort with heavy proteinuria and impaired renal function ${ }^{15}$ to 37.7 weeks in a subgroup with microalbuminuria (IQR 35.6-37.0). ${ }^{12}$ The majority of births reported were premature, ranging from $20 \%$ in a subgroup with microalbuminuria ${ }^{12}$ to $100 \%$ in a cohort with heavy proteinuria and impaired renal function (IQR 43.5-73.9). ${ }^{15}$ Compared with healthy women, DKD was associated with premature delivery (microalbuminuria OR 3.9 (1.5 to 9.90), macroalbuminuria OR 3.9 (1.5 to 9.9)). ${ }^{13}$ One study which included diabetic women with no albuminuria, with microalbuminuria and macroalbuminuria reported premature delivery in $35 \%, 62 \%$ and $91 \%$, respectively. ${ }^{12}$ Very premature births, variably reported as before 32 or 34 weeks, occurred in $0-46 \%$ of births (IQR 9.4-38.6). Compared with healthy women, DKD was associated with very premature delivery (OR 4.2 (1.9 to 9.5)). ${ }^{13}$ The mean birth weight reported ranged from $1880 \mathrm{~g}$ to $3430 \mathrm{~g}$. The $1880 \mathrm{~g}$ occurred in a subgroup with moderately impaired renal function and significant proteinuria ${ }^{5}$ and the $3430 \mathrm{~g}$ occurred in a subgroup with microalbuminuria only. ${ }^{12}$ The ranges for small for gestational age (SGA), where the neonate weighed less than the 10th centile for gestation, and large for gestational age (LGA), where the neonate weighed more than the 90th centile corrected for gestation, varied widely between the stud- ies and were inconsistently reported. The IQR for SGA was 7.7$30.1 \%$ and for LGA was $9.1-33 \%$. One study which included diabetic women with no albuminuria, with microalbuminuria and macroalbuminuria reported rates of SGA in $2 \%, 4 \%$ and $45 \%$, respectively. ${ }^{12}$ Neonatal intensive care unit (NICU) admission was common, reported in $26.2-75 \%$ of births (IQR $41.3-$ 66.8), increased compared with women without DKD (OR 2.4 (1.2 to 4.6$)) .{ }^{13}$ Congenital abnormalities and perinatal deaths were uncommon, reported in 0-14\% (IQR 0-9.2) and 0-14.2\% (IQR 0-9.6), respectively. One study found that diabetic nephropathy (not further characterised) was associated with congenital abnormalities with an adjusted OR of 2.45 (1.14 to 5.25). ${ }^{19}$

Overall higher rates of prematurity, SGA and NICU admissions were noted in the groups with overt proteinuria and impaired renal function than in those with microalbuminuria or normal renal function. Rates were highest where both severe proteinuria and impaired renal function were present.

\section{Blood pressure control}

A number of studies designed to assess the impact of blood pressure on pregnancy outcomes were included. One observational study divided their cohort into two subgroups; one group had a mean arterial blood pressure (MAP) below a target of 100 $\mathrm{mmHg}$ and the other had a MAP of $>100 \mathrm{mmHg}{ }^{5}$ They reported better maternal outcomes (27.3\% pre-eclampsia versus $42 \%$ ) and fetal outcomes (mean gestation 35.1 weeks versus 32.1 weeks) in the target MAP group. ${ }^{5}$ Two further studies ${ }^{12,20}$ reported an improvement in maternal and fetal outcomes with more intensive control of hypertension.

\section{Renal outcomes (Table 5)}

Only two of the papers published in the last 20 years reported on longer term renal outcomes. One paper, which followed 14 women with albuminuria $>300 \mathrm{mg}$ at the time of pregnancy for a mean of 6 years, reported $36 \%$ reached end-stage renal failure in that time. There was no control group. ${ }^{21}$ The other paper followed 26 women with diabetic nephropathy who had pregnancies and 67 women with diabetic nephropathy without pregnancies for 10 years. The outcomes were similar in both groups, with a slightly higher incidence of end-stage renal failure in the group without pregnancy. ${ }^{22}$

\section{Discussion}

This systematic review of pregnancy outcomes and DKD showed that most women were relatively young, nulliparous and had a long duration of diabetes, usually type 1 . There were high rates of maternal and fetal complications and these were more common in women with macroalbuminuria or impaired renal function. For comparison, in the general population pre-eclampsia affects $5 \%$ of women, $7.3 \%$ of babies arrive preterm (prior to 37 weeks), ${ }^{23} 77 \%$ of birth weights are $>3000 \mathrm{~g}^{24}$ and $10.9-$ $14.5 \%$ of babies are admitted to the NICU..$^{25}$ This review highlights high rates of Caesarean section in women with DKD. Women with diabetes already have higher rates of Caesarean 
Table 3 Maternal outcomes

\begin{tabular}{|c|c|c|c|c|c|c|}
\hline & $\begin{array}{l}\text { Pre-eclampsia } \\
(\%)\end{array}$ & $\begin{array}{l}\text { Caesarean } \\
\text { section (\%) }\end{array}$ & $\begin{array}{l}\text { Maternal } \\
\text { deaths (\%) }\end{array}$ & $\begin{array}{l}\text { Dialysis during } \\
\text { pregnancy (\%) }\end{array}$ & $\begin{array}{l}\text { Miscarriage } \\
(\%)\end{array}$ & $\begin{array}{l}\text { Abortion } \\
(\%)\end{array}$ \\
\hline Reece, $1990^{19}$ & NR & $N R$ & $N R$ & NR & Ex & Ex \\
\hline Combs, $1993^{20}$ & 47 & NR & $N R$ & NR & Ex & Ex \\
\hline Hod, $1995^{21}$ & 38 & 75 & 0 & 0 & Ex & Ex \\
\hline $\begin{array}{l}\text { Kimmerle, } 1995^{4} \\
\text { Preserved renal function } \\
\text { Non-preserved renal function }\end{array}$ & $N R$ & $\begin{array}{l}80 \\
100\end{array}$ & $N R$ & $N R$ & 0 & 10 \\
\hline Gordon, $1996^{22}$ & 53 & 80 & $N R$ & $N R$ & 7.8 & 3.9 \\
\hline Kaaja, $1996^{23}$ & NR & NR & $N R$ & NR & NR & NR \\
\hline $\begin{array}{l}\text { Mackie, } 1996^{10} \\
\text { Moderate renal impairment } \\
\text { Mild renal impairment }\end{array}$ & $N R$ & $\begin{array}{l}100 \\
100\end{array}$ & $N R$ & $N R$ & $\begin{array}{l}27 \\
0\end{array}$ & $\begin{array}{l}9 \\
7\end{array}$ \\
\hline Miodovnik, 199624 & 76 & 76 & NR & NR & Ex & Ex \\
\hline Purdy, 199625 & NR & NR & $\mathrm{NR}$ & NR & NR & NR \\
\hline Zhu, $1997^{26}$ & 40 & 90 & $N R$ & $N R$ & NR & NR \\
\hline Reece, $1998^{27}$ & 53 & 63 & $N R$ & NR & NR & NR \\
\hline Bar, $1999^{28}$ & 46 & 62.5 & NR & NR & Ex & Ex \\
\hline Biesenbach, $1999^{11}$ & 57.1 & 50 & NR & NR & Ex & Ex \\
\hline Dunne, $1999^{29}$ & 50 & 90.5 & NR & NR & Ex & Ex \\
\hline Biesenbach, $2000^{30}$ & 60 & 60 & $N R$ & $N R$ & NR & NR \\
\hline $\begin{array}{l}\text { Ekbom, } 2001^{14} \\
\text { DN } \\
\text { Microalbuminuria }\end{array}$ & $\begin{array}{l}42 \\
64\end{array}$ & NR & NR & NR & NR & NR \\
\hline $\begin{array}{l}\text { Khoury, } 2002^{9} \\
\mathrm{Cr}<1 \mathrm{mg} / \mathrm{dL} \\
\mathrm{Cr} 1-1.5 \mathrm{mg} / \mathrm{dL} \\
\mathrm{Cr}>1.5 \mathrm{mg} / \mathrm{dL}\end{array}$ & $\begin{array}{l}41 \\
33.3 \\
44.4\end{array}$ & $\begin{array}{l}76.9 \\
91.7 \\
88.9\end{array}$ & 0 & 0 & $\begin{array}{l}49 \\
13 \\
10 \%\end{array}$ & NR \\
\hline Rossing, $2002^{18}$ & 41 & 38.7 & 0 & 0 & Ex & Ex \\
\hline Bagg, $2003^{31}$ & $\mathrm{NR}$ & 83 & NR & NR & NR & NR \\
\hline $\begin{array}{l}\text { Carr, } 2006^{7} \\
\text { Above target BP (MAP >100 mmHg) } \\
\text { Below target (MAP }<100 \mathrm{mmHg} \text { ) }\end{array}$ & $\begin{array}{l}27.3 \\
42.9\end{array}$ & $\begin{array}{l}63.4 \\
76.2\end{array}$ & 0 & 0 & Ex & Ex \\
\hline Nielson, $2006^{32}$ & 42 & 20 & & & & \\
\hline $\begin{array}{l}\text { Nielsen, } 2009^{12} \\
\text { Diabetic nephropathy } \\
\text { Microalbuminuria }\end{array}$ & $\begin{array}{l}43 \\
0\end{array}$ & $N R$ & $N R$ & $N R$ & Ex & Ex \\
\hline $\begin{array}{l}\text { Yogev, } 2009^{33} \\
\text { Non-complicated pregnancy } \\
\text { Complicated pregnancy }\end{array}$ & NR & $\begin{array}{l}67 \\
78\end{array}$ & $N R$ & $N R$ & $\begin{array}{l}0 \\
10\end{array}$ & 0 \\
\hline Jensen, $2010^{34}$ & 41 & NR & $N R$ & NR & Ex & Ex \\
\hline Bell, $2012^{17}$ & NR & NR & NR & NR & $x$ & NR \\
\hline Young, $2012^{35}$ & 63.6 & $N R$ & 0 & NR & Ex & Ex \\
\hline $\begin{array}{l}\text { Damm, } 2013^{5} \\
\text { Type } 2 \text { DN } \\
\text { Type } 1 \text { DN } \\
\text { T2 microalbuminuria } \\
\text { T1 microalbuminuria }\end{array}$ & $\begin{array}{l}40 \\
36 \\
10 \\
20\end{array}$ & $\begin{array}{l}60 \\
91 \\
80 \\
80\end{array}$ & NR & $\begin{array}{l}0 \\
0 \\
0 \\
0\end{array}$ & Ex & Ex \\
\hline Piccoli, $2013^{15}$ & NR & $75 \%$ & 0 & 0 & Ex & Ex \\
\hline $\begin{array}{l}\text { Klemetti, } 2015^{16} \\
1988-1999 \text { group } \\
\text { 2000-2011 group }\end{array}$ & $\begin{array}{l}52.3 \\
41.9\end{array}$ & $\begin{array}{l}100 \\
92.9\end{array}$ & $N R$ & $1 \%$ & Excluded & Excluded \\
\hline $\begin{array}{l}\text { Seah, } 2020^{13} \\
\text { Microalbuminuria } \\
\text { Macroalbuminuria }\end{array}$ & $\begin{array}{l}\text { OR } 5.7 \text { ( } 1.8 \text { to } 17.8) \\
\text { OR } 5.5 \text { ( } 2.5 \text { to } 11.8)\end{array}$ & NR & NR & NR & Ex & Ex \\
\hline
\end{tabular}


Table 4. Fetal outcomes

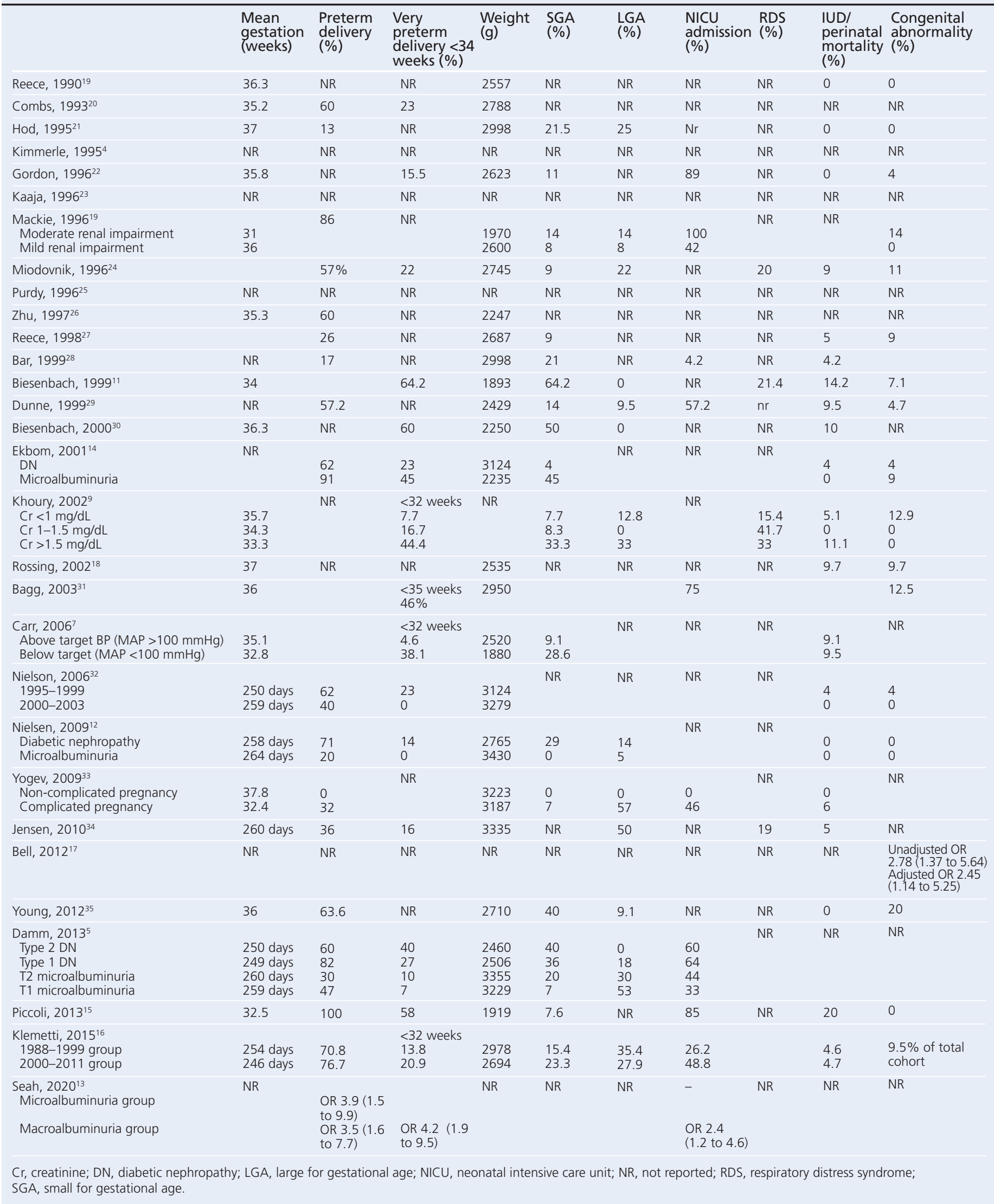


Table 5 Long-term renal outcomes

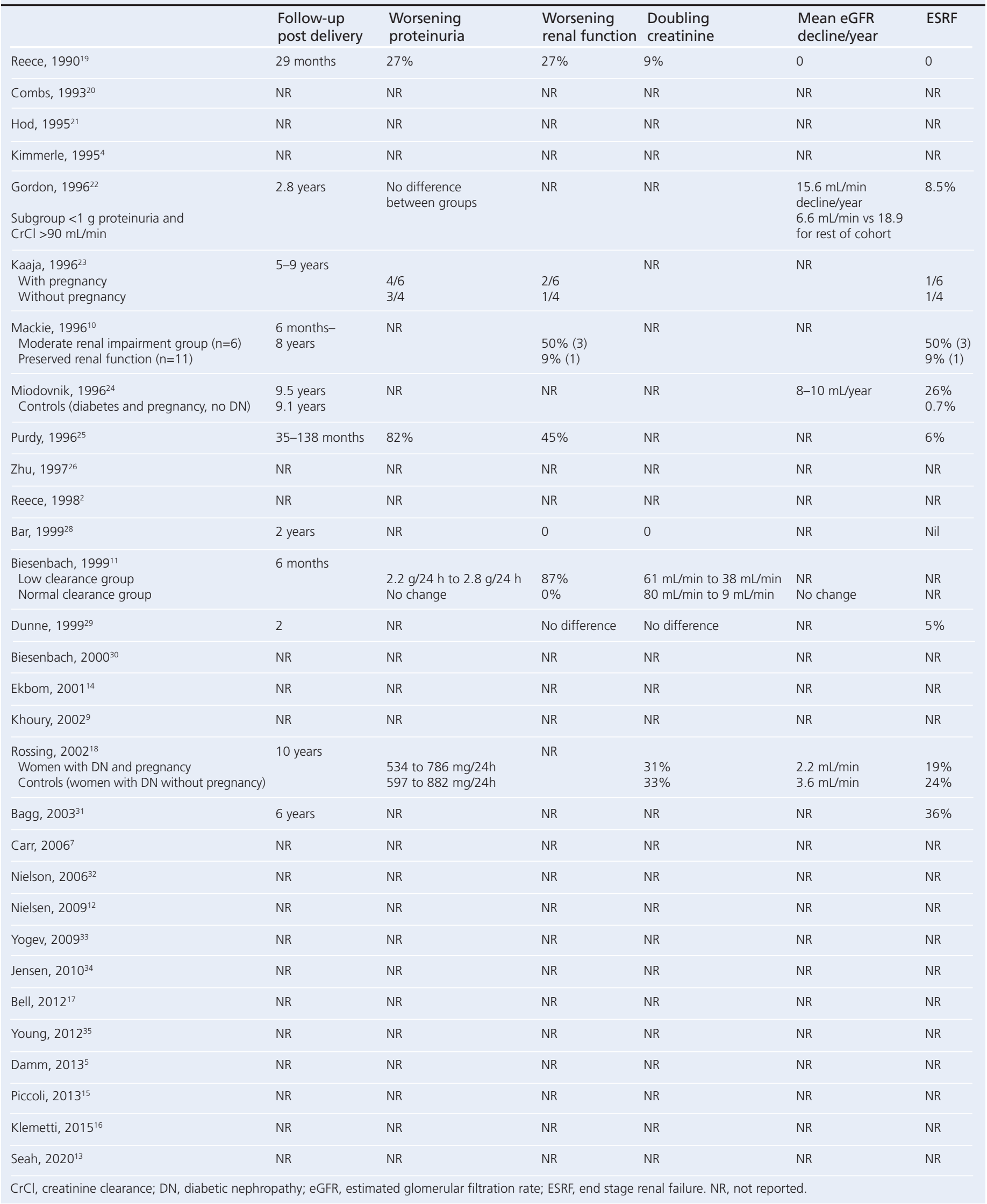


Table 6 Management of diabetic kidney disease in pregnancy

\begin{tabular}{|c|c|}
\hline Pre-pregnancy & $\begin{array}{l}\text { - Women with diabetes should have an assessment of their renal function (including proteinuria) prior to stopping contraception } \\
\text { - Women with a creatinine }>120 \mathrm{mmol} / \mathrm{L} \text {, albuminuria }>30 \mathrm{mg} / \mathrm{mmol} \text { or eGFR }<45 \mathrm{~mL} / \mathrm{min} \text { should be referred to a nephrologist prior to } \\
\text { pregnancy } \\
\text { Women with diabetic nephropathy should be offered pre-pregnancy counselling to inform them of potential adverse pregnancy outcomes } \\
\text { and to allow optimisation of blood pressure, glycaemic control and proteinuria prior to pregnancy } \\
\text { They should remain on angiotensin converting enzyme inhibitors until conception, with regular pregnancy testing during attempts to } \\
\text { conceive } \\
\text { - The } \mathrm{HbA} \text { c should be below } 48 \mathrm{mmol} / \mathrm{mol} \text { prior to conception (if achievable without causing problematic hypoglycaemia) } \\
\text { - High dose folic acid } 5 \mathrm{mg} \text { should be started } 3 \text { months prior to conception }\end{array}$ \\
\hline During pregnancy & $\begin{array}{l}\text { - Women with a creatinine }>120 \mathrm{mmol} / \mathrm{L} \text {, albuminuria }>30 \mathrm{mg} / \mathrm{mmol} \text { or PCR }>50 \mathrm{mg} / \mathrm{mmol} \text { should see a nephrologist during pregnancy } \\
\text { (Note: eGFR should not be used during pregnancy) } \\
\text { - They should have regular MDT visits throughout gestation (every } 1-2 \text { weeks) } \\
\text { - They should be offered low-dose aspirin ( } 75-150 \mathrm{mg} \text { ) before } 16 \text { weeks of gestation as pre-eclampsia prophylaxis } \\
\text { - Women with nephrotic range proteinuria (PCR }>300 \mathrm{mg} / \mathrm{mmol} \text { or } \mathrm{ACR}>250 \mathrm{mg} / \mathrm{mmol} \text { should be offered prophylactic low molecular } \\
\text { - Target blood pressure of } 110-130 \mathrm{mmHg} \text { (systolic) and } 70-90 \mathrm{mmHg} \text { (diastolic) should be used } \\
\text { - The creatinine and ACR/PCR should be checked at least 4-weekly and at least fortnightly from } 32 \text { weeks of gestation }\end{array}$ \\
\hline Post-partum & $\begin{array}{l}\text { - Restart RAAS blockade post-partum as soon as renal function is stable. In breastfeeding, enalapril and captopril are the preferred ACE } \\
\text { inhibitors, and angiotensin receptor blockade is not advised until breastfeeding cessation } \\
\text { - } \quad \text { Ensure follow-up with nephrologist post-partum (and with the diabetes services if not already engaged) }\end{array}$ \\
\hline
\end{tabular}

section than the general population ( $46 \%$ versus $12 \%){ }^{3}$ This risk is higher again in women with DKD. The additive risks of pre-eclampsia, growth restriction and concern over loss of kidney function likely contribute to the high rate of prematurity.

Historically, women with diabetic nephropathy had high rates of fetal loss, obstetric complications and progression to end-stage renal failure in pregnancy. In recent years, with improved diagnosis and management of DKD before and during pregnancy, outcomes have improved. However, the risk of complications is much higher than in healthy women and women with diabetes without kidney disease, as detailed above. The papers included in this review have informed our current knowledge and have been incorporated in a number of comprehensive guidelines including the National Institute for Health and Care Excellence and American Diabetes Association guidelines on management of diabetes in pregnancy and the Renal Association guidelines on Pregnancy and Renal Disease..$^{26-28}$ Important aspects of management include pre-pregnancy counselling, close multidisciplinary antenatal monitoring with strict blood pressure control, pre-eclampsia prophylaxis and consideration of thromboprophylaxis and early reintroduction of ACE inhibitors and ensuring appropriate follow-up postnatally. Key management points are summarised in Table 6.

This systematic review was limited by the quality of the studies included; they were most often retrospective, small and monocentric and may have been subject to selection or reporting biases. As a result of these very heterogeneous studies, the results reported varied widely between studies. The variations in the definition of DKD used, the evolving definition of preeclampsia and the notorious difficulty diagnosing pre-eclampsia in women with pre-existing hypertension and proteinuria are likely also to have affected the reported outcomes. As diabetes and DKD are common conditions, it is vital for women and their doctors from different disciplines, including obstetrics, endocrinology and nephrology, to be fully aware of the risks asso-

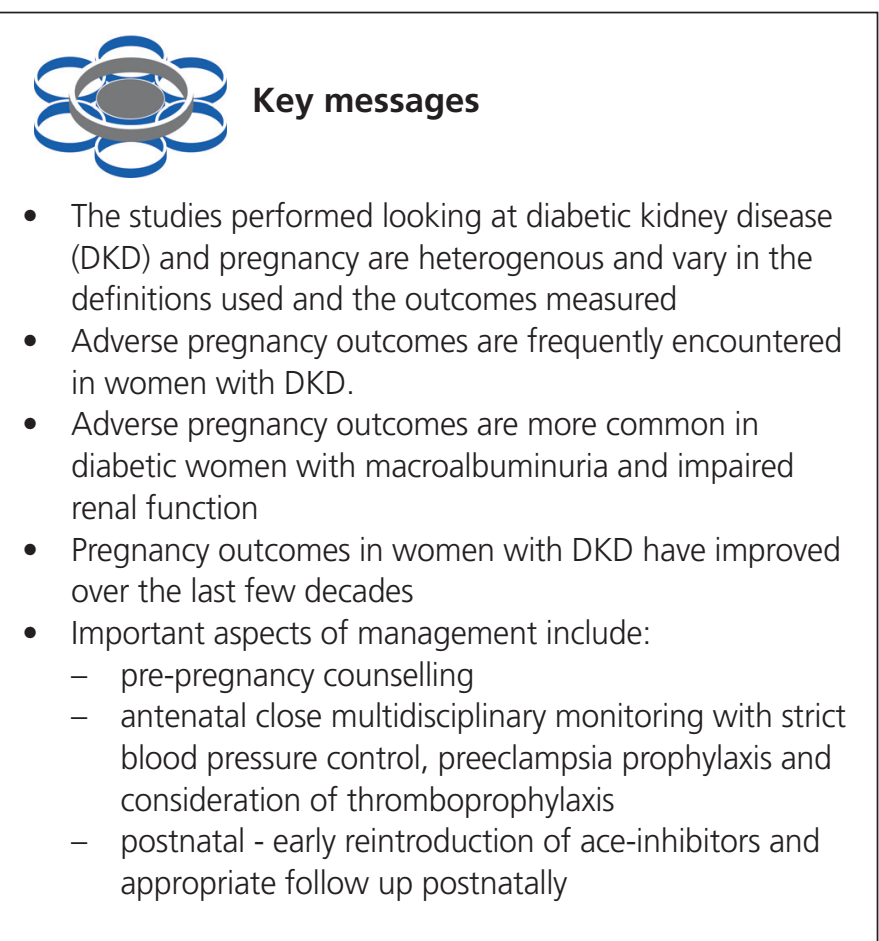

ciated with pregnancy. This will empower women to make a fully informed decision when considering pregnancy and enable better obstetric and renal care, leading to a safer pregnancy with better outcomes.

\section{Conflict of interest None. Funding None.}

\section{References}

1. Confidential Enquiry into Maternal and Child Health. Diabetes in pregnancy: are we providing the best care? Findings of a national enquiry. 
England, Wales and Northern Ireland. London: Confidential Enquiry into Maternal and Child Health, 2007.

2. Ringholm L, Damm JA, Vestgaard M, Damm P, Mathiesen ER. Diabetic nephropathy in women with preexisting diabetes: from pregnancy planning to breastfeeding. Curr Diab Rep 2016;16(2):12. https://doi.org/ 10.1007/s11892-015-0705-3

3. Persson M, Norman M, Hanson U. Obstetric and perinatal outcomes in type 1 diabetic pregnancies: a large, population-based study. Diabetes Care 2009;32(11):2005-9. https://doi.org/10.2337/dc09-0656

4. White P. Pregnancy complicating diabetes. J Am Med Assoc 1945; 128(3):181-2.

5. Carr D, Koontz G, Gardella C, et al. Diabetic nephropathy in pregnancy: suboptimal hypertensive control associated with preterm delivery. Am J Hypertens 2006;19(5):513-19. https://doi.org/10.1016/ j.amjhyper.2005.12.010

6. Kimmerle R, Zass RP, Cupisti S, et al. Pregnancies in women with diabetic nephropathy: long-term outcome for mother and child. Diabetologia 1995;38(2):227-35.

7. Bramham K. Diabetic nephropathy and pregnancy. Semin Nephrol 2017;37(4):362-9. https://doi.org/10.1016/j.semnephrol.2017.05.008

8. Ekbom P. Pre-pregnancy microalbuminuria predicts pre-eclampsia in insulin-dependent diabetes mellitus. Lancet 1999;353(9150):377. https://doi.org/10.1016/s0140-6736(05)74949-7

9. Moher D, Shamseer L, Clarke M, et al. Preferred reporting items for systematic review and meta-analysis protocols (PRISMA-P) 2015 statement. Syst Rev 2015;4(1):1.

10. Khoury JC, Miodovnik M, LeMasters G, Sibai B. Pregnancy outcome and progression of diabetic nephropathy. What's next? J Matern Fetal Neonatal Med 2002;11:238-44. https://doi.org/10.1080/ jmf.11.4.238.244

11. Damm JA, Asbjornsdottir B, Callesen NF, et al. Diabetic nephropathy and microalbuminuria in pregnant women with type 1 and type 2 diabetes: prevalence, antihypertensive strategy, and pregnancy outcome. Diabetes Care 2013;36(11):3489-94. https://doi.org/10.2337/dc131031

12. Nielsen LR, Damm P, Mathiesen ER. Improved pregnancy outcome in type 1 diabetic women with microalbuminuria or diabetic nephropathy: effect of intensified antihypertensive therapy? Diabetes Care 2009; 32(1):38-44. https://doi.org/10.2337/dc08-1526

13. Seah J, Kam NM, Wong L, et al. The association between maternal renal function and pregnancy outcomes in type 1 and type 2 diabetes. Diabetes Res Clin Pract 2020;165:108225. https://doi.org/10.1016/j.diabres.2020.108225

14. Ekbom P, Damm P, Feldt-Rasmussen B, Feldt-Rasmussen U, Mølvig J, Mathiesen ER. Pregnancy outcome in type 1 diabetic women with microalbuminuria. Diabetes Care 2001;24(10):1739-44. https://doi.org/ 10.2337/diacare.24.10.1739

15. Piccoli GB, Tavassoli E, Melluzza C, et al. Severe diabetic nephropathy in type 1 diabetes and pregnancy - a case series. Rev Diabet Stud 2013:10(1):68-78. https://doi.org/10.1900/RDS.2013.10.68
16. Yogev Y, Chen R, Ben-Haroush A, Hod M, Bar J. Maternal overweight and pregnancy outcome in women with type-1 diabetes mellitus and different degrees of nephropathy. I Matern Fetal Neonatal Med 2010;23(9):999-1003. https://doi.org/10.3109/14767050903544744

17. Young EC, Pires MLE, Marques LPJ, de Oliveira JEP, Zajdenverg L. Effects of pregnancy on the onset and progression of diabetic nephropathy and of diabetic nephropathy on pregnancy outcomes. Diabetes Metab Syndr 2011;5(3):137-42. https://doi.org/10.1016/j.dsx.2012.02.013

18. Klemetti MM, Laivuori $H$, Tikkanen $M$, Nuutila M, Hiilesmaa V, Teramo K. Obstetric and perinatal outcome in type 1 diabetes patients with diabetic nephropathy during 1988-2011. Diabetologia 2015;58(4):67886. https://doi.org/10.1007/s00125-014-3488-1

19. Bell R, Glinianaia SV, Tennant PWG, Bilous RW, Rankin J. Peri-conception hyperglycaemia and nephropathy are associated with risk of congenital anomaly in women with pre-existing diabetes: a population-based cohort study. Diabetologia 2012;55(4):936-47. https://doi.org/10.1007/ s00125-012-2455-y

20. Nielsen LR, Muller C, Damm P, Mathiesen ER. Reduced prevalence of early preterm delivery in women with type 1 diabetes and microalbuminuria-possible effect of early antihypertensive treatment during pregnancy. Diabet Med 2006;23(4):426-31. https://doi.org/10.1111/ j.1464-5491.2006.01831.x

21. Bagg W, Neale L, Henley P, MacPherson P, Cundy T. Long-term maternal outcome after pregnancy in women with diabetic nephropathy. $N Z$ Med J 2003;116(1180):U566.

22. Rossing $\mathrm{K}$, Jacobsen $\mathrm{P}$, Hommel E, et al. Pregnancy and progression of diabetic nephropathy. Diabetologia 2002;45(1):36-41. https://doi.org/ $10.1007 / s 125-002-8242-4$

23. National Institute for Health and Care Excellence (NICE). Preterm labour and birth. NICE Guideline [NG25]. 2015 (updated 2019). Available from: https://www.nice.org.uk/guidance/ng25/chapter/context

24. NHS Digital. NHS maternity statistics, England 2019-20. Available from: https://digital.nhs.uk/data-and-information/publications/statistical/nhsmaternity-statistics/2019-20

25. Braun D, Braun E, Chiu V, et al. Trends in Neonatal intensive care unit utilization in a large integrated health care system. JAMA Netw Open [Internet]. 2020;3(6):e205239. https://doi.org/10.1001/jamanetworkopen.2020.5239

26. National Institute for Health and Care Excellence (NICE). Diabetes in pregnancy: management from preconception to the postnatal period. NICE Guideline [NG3]. 2015 (updated 2020). Available from: https://www.nice.org.uk/guidance/ng3

27. Wiles K, Chappell L, Clark K, et al. Clinical practice guideline on pregnancy and renal disease. BMC Nephrol 2019;20(1):401. https://doi.org/10.1186/s12882-019-1560-2

28. American Diabetes Association. 14. Management of Diabetes in Pregnancy: standards of medical care in diabetes -2020 . Diabetes Care 2020;43(Suppl 1):S183-92. https://doi.org/10.2337/dc20-S014 